\title{
Gamma-ray observations of Nova Sgr 2015 No. 2 with INTEGRAL
}

\author{
Thomas Siegert ${ }^{1,2}$, Alain Coc $^{3}$, Laura Delgado ${ }^{4,5}$, Roland Diehl ${ }^{1,2}$, Jochen Greiner ${ }^{1}$, Margarita Hernanz ${ }^{4,5}$, \\ Pierre Jean $^{6}$, Jordi José ${ }^{7,5}$, Paolo Molaro ${ }^{8}$, Moritz M. M. Pleintinger ${ }^{1}$, Volodymyr Savchenko ${ }^{9}$, \\ Sumner Starrfield ${ }^{10}$, Vincent Tatischeff ${ }^{3}$, and Christoph Weinberger ${ }^{1}$ \\ ${ }^{1}$ Max-Planck-Institut für extraterrestrische Physik, Gießenbachstraße, 85741 Garching, Germany \\ e-mail: tsiegert@mpe.mpg.de \\ ${ }^{2}$ Excellence Cluster Universe, Boltzmannstraße 2, 85748 Garching, Germany \\ ${ }^{3}$ Centre de Sciences Nucléaires et de Sciences de la Matière (CSNSM), CNRS/IN2P3, Univ. Paris-Sud, Université Paris-Saclay, \\ 91405 Orsay Campus, France \\ ${ }^{4}$ Institute of Space Science (ICE, CSIC), Campus UAB, C/ Can Magrans s/n, 08193 Cerdanyola del Valles (Barcelona), Spain \\ ${ }^{5}$ Institut d'Estudis Espacials de Catalunya (IEEC), 08034 Barcelona, Spain \\ ${ }^{6}$ CNRS, IRAP, 9 avenue du Colonel Roche, BP 44346, 31028 Toulouse Cedex 4, France \\ ${ }^{7}$ Departament de Física, Universitat Politècnica de Catalunya, BarcelonaTech, EEBE, C/ Eduard Maristany 10, 08019 Barcelona, \\ Spain \\ ${ }^{8}$ INAF, Osservatorio Astronomico di Trieste, Via G. B. Tiepolo 11, 34131 Trieste, Italy \\ 9 ISDC, Department of astronomy, University of Geneva, chemin d'Ecogia, 161290 Versoix, Switzerland \\ ${ }^{10}$ School of Earth and Space Exploration, Arizona State University, Tempe, Arizona 85287 1404, USA
}

Received 21 December 2017 / Accepted 15 March 2018

\begin{abstract}
Context. INTEGRAL observed Nova Sgr 2015 No. 2 (V5668 Sgr) around the time of its optical emission maximum on 21 March 2015. Studies at UV wavelengths showed spectral lines of freshly produced ${ }^{7} \mathrm{Be}$. This could also be measurable in gamma rays at $478 \mathrm{keV}$ from the decay to ${ }^{7} \mathrm{Li}$. Novae are also expected to synthesise ${ }^{22} \mathrm{Na}$ which decays to ${ }^{22} \mathrm{Ne}$, emitting a $1275 \mathrm{keV}$ photon. About one week before the optical maximum, a strong gamma-ray flash on timescales of hours is expected from short-lived radioactive nuclei such as ${ }^{13} \mathrm{~N}$ and ${ }^{18} \mathrm{~F}$. These nuclei are $\beta^{+}$-unstable, and should yield emission of up to $511 \mathrm{keV}$, but this emission has never been observed from any nova.

Aims. The SPectrometer on INTEGRAL (SPI) pointed towards V5668 Sgr by chance. We use these observations to search for possible gamma-ray emission of decaying ${ }^{7} \mathrm{Be}$, and to directly measure the synthesised mass during explosive burning. We also aim to constrain possible burst-like emission days to weeks before the optical maximum using the SPI anticoincidence shield (ACS), i.e. at times when SPI was not pointing to the source.

Methods. We extracted spectral and temporal information to determine the fluxes of gamma-ray lines at $478 \mathrm{keV}, 511 \mathrm{keV}$, and $1275 \mathrm{keV}$. Using distance and radioactive decay, a measured flux converts into the ${ }^{7} \mathrm{Be}$ amount produced in the nova. The SPI-ACS rates are analysed for burst-like emission using a nova model light curve. For the obtained nova flash candidate events, we discuss possible origins using directional, spectral, and temporal information.

Results. No significant excess for the $478 \mathrm{keV}$, the $511 \mathrm{keV}$, or the $1275 \mathrm{keV}$ lines is found. Our upper limits $(3 \sigma)$ on the synthesised ${ }^{7} \mathrm{Be}$ and ${ }^{22} \mathrm{Na}$ mass depend on the uncertainties of the distance to V5668 Sgr: the ${ }^{7} \mathrm{Be}$ mass is constrained to less than $4.8 \times 10^{-9}\left(\mathrm{dkpc}^{-1}\right)^{2} M_{\odot}$, and the ${ }^{22} \mathrm{Na}$ mass to less than $2.4 \times 10^{-8}\left(\mathrm{dkpc}^{-1}\right)^{2} M_{\odot}$. For the ${ }^{7} \mathrm{Be}$ mass estimate from UV studies, the distance to V5668 Sgr must be greater than $1.2 \mathrm{kpc}(3 \sigma)$. During the three weeks before the optical maximum, we find 23 burst-like events in the ACS rate, of which 6 could possibly be associated with V5668.
\end{abstract}

Key words. novae, cataclysmic variables - white dwarfs - gamma rays: general - nuclear reactions, nucleosynthesis, abundances techniques: spectroscopic

\section{Introduction}

On 15 March 2015, Nova Sagittarii 2015 No. 2 (V5668 Sgr, or V5668) was detected by Seach (2015) at galactic coordinates $\left(l_{0} / b_{0}\right)=\left(5.38^{\circ} /-9.87^{\circ}\right)$. After a six-day rise in brightness, V5668 reached its optical maximum on March 21.67 UT, corresponding to $T_{0}=$ MJD 57102.67 , with a $V$-band magnitude of $4.32 \mathrm{mag}$. Two independent studies (Molaro et al. 2016; Tajitsu et al. 2016) measured blue-shifted spectral lines of singly ionised Be II at wavelengths around $313 \mathrm{~nm}$. The Doppler velocities of these UV line profiles range between -700 and $-2200 \mathrm{~km} \mathrm{~s}^{-1}$.
Based on a canonical ejected mass of $10^{-5} M_{\odot}$ for novae, the measured abundance ratios allowed the authors to estimate the mass of synthesised and ejected ${ }^{7} \mathrm{Be}$ for V5668 from these UV measurements of $7 \times 10^{-9} M_{\odot}$ (Molaro et al. 2016). Novae have only recently been verified as significant sources of ${ }^{7} \mathrm{Li}$ in the Galaxy by detections of ${ }^{7} \mathrm{Li}$ I at $6708 \AA$ in Nova Centauri 2013 (V1369 Cen, Izzo et al. 2015), and the ${ }^{7}$ Be II doublet at $313.0583 \mathrm{~nm}$ and $313.1228 \mathrm{~nm}$ in Nova Delphini 2013 (V339 Del; Tajitsu et al. 2015).

The distance to V5668 is not precisely known. Based on expansion velocity measurements and geometrical 
considerations, Banerjee et al. (2016) estimated a distance ${ }^{1}$ of $1.54 \mathrm{kpc}$. Jack et al. (2017) derived a similar estimate of $1.6 \mathrm{kpc}$, using a distance modulus approach, but without providing uncertainties. The maximum magnitude vs. rate decline (MMRD) method for determining the distance to novae, as reviewed by (della Valle \& Livio 1995; see also Schmidt 1957), provides similar distance estimates but with an intrinsically large uncertainty. Because the luminosity of a nova outburst depends on more physical parameters than only the white dwarf mass (della Valle \& Livio 1995), the uncertainty on the distance to a single nova, as opposed to a population, might be as high as $\approx 50 \%$.

V5668 Sgr has been observed at many wavelengths. In midinfrared (e.g. Gehrz et al. 2015, with SOFIA/FORCAST) and near-infrared (e.g. Banerjee et al. 2016, using NICS) observations, clear signatures of dust have been seen, and a strong detection of CO in emission as well (Banerjee et al. 2015). The total dust mass produced by V5668 is estimated to be about $2.7 \times 10^{-7} M_{\odot}$ (Banerjee et al. 2016), so that the mass of the gaseous component of the ejecta is between 2.7 and $5.4 \times 10^{-5} M_{\odot}$. We note that the ejecta mass is difficult to estimate because the absolute flux measurements have to be scaled by the distance to a nova, which is model-dependent and often has uncertainties of several tens of percent, and also because there is no adequate observable to estimate the accretion rate. In the optical, V5668 was monitored for more than 200 days after the outburst (Jack et al. 2017, using TIGRE); transient Balmer and Paschen lines of $\mathrm{H}$, different Fe II lines, and N I and N II lines were found. In general, the spectral shapes change during the evolution of the optical light curve, whereas clear double-peak profiles are observed after the deep minimum at day 110 after the optical maximum. The spread in Doppler velocities (expanding shell velocity) is up to $2000 \mathrm{~km} \mathrm{~s}^{-1}$. About 95 days after the outburst, the nova was detected in soft X-rays (Page et al. 2015, with Swift/XRT), softening and brightening up to $(6.0 \pm 0.5) \times 10^{-2} \mathrm{cts} \mathrm{s}^{-1}$ until day 161. During this time, the apparent H-column density towards V5668 decreased from $N_{H}=\left(4.7_{-1.2}^{+1.6}\right) \times 10^{22} \mathrm{~cm}^{-1}$ to $(0.4 \pm 0.1) \times 10^{22} \mathrm{~cm}^{-1}$, while the plasma temperature increased from $1.3_{-0.3}^{+0.5} \mathrm{keV}$ to $3.4_{-0.8}^{+1.4} \mathrm{keV}$. This spectral change may be connected to the destruction of dust by soft X-ray and UV emission (Page et al. 2015, Swift/UVOT).

High-energy gamma rays have also been observed for V5668 (0.1-100 GeV; Cheung et al. 2016, using Fermi/LAT) beginning about two days after the optical maximum, as measured from similar nova outbursts (e.g. Hays et al. 2013; Cheung et al. 2013; Ackermann et al. 2014). In the $>100 \mathrm{MeV}$ band, V5668 was visible for 55 days with an average flux of $\approx 10^{-7} \mathrm{ph} \mathrm{cm}^{-2} \mathrm{~s}^{-1}$, fainter than observed for this type of source. We note that fewer than ten gamma-ray novae have been detected prior to the write-up of this paper (Ackermann et al. 2014). Even though the gamma-ray emission appears sporadic and may be delayed to the optical emission, it seems that the hard gammaray flux correlates with the optical light curve. In fact, for the brightest gamma-ray nova detected to date, ASASSN-16ma (Luckas 2016), the optical light curve strongly correlates with the 0.1-300 GeV flux during the decline phase. The ratio of gamma ray to optical flux remained constant at a value of $\approx 0.002$.

\footnotetext{
1 Banerjee et al. (2016) provide three distance estimates, one that considers the geometry and expansion velocity but without uncertainties, and two others based on two different MMRD method assumptions, leading to two different absolute magnitudes, $M_{V}=-6.91 \pm 0.40$ and $-6.65 \pm 1.82$, and thus two distances of $1.31-1.76$ and $0.68-3.6 \mathrm{kpc}$, respectively.
}

This tight correlation made the authors cast doubt on the standard model for optical nova emission. Nuclear burning on the surface of the white dwarf (see Sect. 1) results in freshly synthesised nuclei, which then are ejected, decay, and/or de-excite and emit $\mathrm{MeV}$ gamma rays. Some of these gamma rays may undergo Compton scattering, which powers a continuum with a low-energy cut-off around $20-30 \mathrm{keV}$ due to photo-electric absorption (Gomez-Gomar et al. 1998). The optical emission, on the other hand, is mainly thermal radiation from the heated gas, which is now cooling because it is expanding. Even though the nova explosion is triggered by nucleosynthesis reactions, the resulting $\sim \mathrm{MeV}$ gamma rays only provide a small amount of energy during the envelope expansion. The visual maximum corresponds, therefore, to the maximum expansion of the photosphere. After the optical peak the photosphere recedes, and higher temperatures become visible so that the peak moves to UV wavelengths. However, this scenario cannot explain the highenergy GeV gamma-ray emission, which is generally attributed to shock-accelerated particles, and expecially cannot explain the correlation between the optical and $\mathrm{GeV}$ emission. Instead, the authors propose that the optical emission predominantly originates in the shocks rather than in the photosphere above. This automatically explains the simultaneous emission and also the questionable super-Eddington luminosities observed for many novae because shocks may not be treated as hydrostatic atmospheres (see also Martin et al. 2018). In the case of V5668, the Fermi/LAT $>100 \mathrm{MeV}$ light curve also seems to show multiple peaks, which means that this nova might have had multiple mass ejections, and thus there might be multiple onsets of nucleosynthesis. If a local maximum in the optical or $>100 \mathrm{MeV}$ light curve indeed corresponds to an additional mass ejection, the estimates of this and other works might be more uncertain, as each individual outburst might eject its own amount of mass. In this paper, we focus on a single time origin of explosive burning, and discuss only one major mass ejection.

This shock scenario also predicts hard X-ray emission at later times, contemporaneous to the $\mathrm{GeV}$ emission, depending on the nova outflow properties such as density, mass, velocity, and the resulting optical depth. The peak of such an additional X-ray emission would be expected between 30 and 210 days after the optical maximum (Metzger et al. 2014). These non-thermal $\mathrm{X}$-rays would be produced by optical $(\mathrm{eV})$ photons, being Compton up-scattered on $\mathrm{GeV}$ particles. Following Metzger et al. (2015), an order of magnitude estimate for the expected $\mathrm{X}$-ray flux can be derived from the measured $\mathrm{GeV}$ flux $F_{\mathrm{GeV}}$. This assumes a fraction $f_{X}$ of high-energy gamma-ray luminosity to be radiated away in X-rays of energy $E_{X}$, so that the resulting $\mathrm{X}$-ray flux is $F_{X} \sim f_{X} F_{\mathrm{GeV}} / E_{X}$. For example, an $f_{X}$ value of 0.01 would predict a hard X-ray flux of the order of $10^{-6} \mathrm{ph} \mathrm{cm}^{-2} \mathrm{~s}^{-1} \mathrm{keV}^{-1}$ for V5668 Sgr at about $50 \mathrm{keV}$. The influence of such a plausible additional flux at hard X-rays and also soft gamma rays on the INTEGRAL measurements is discussed in Sect. 3.1.

The nova explosion is typically explained by a thermonuclear runaway on the surface of a white dwarf. At a mass accretion rate of $10^{-10}-10^{-9} M_{\odot} \mathrm{yr}^{-1}$, the accreted matter reaches degeneracy by the strong gravitational field of the white dwarf. Once the ignition conditions for hydrogen burning are met, nucleosynthesis starts. Even though the envelope is initially degenerate, once the temperature in the envelope exceeds $3 \times 10^{7} \mathrm{~K}$, degeneracy is lifted in the whole envelope (José 2016). However, it is important to stress that a nova outburst likely occurs because of a hydrogen thin shell instability (Schwarzschild \& Härm 1965; Yoon et al. 2004), for which degeneracy is not required at all. 
In general, nuclei up to $A \approx 40$ are produced and ejected in a classical nova explosion. In this scenario, hydrogen burning proceeds through the $\mathrm{CNO}$-cycle, i.e. it is required that such seed nuclei are present. During the CNO burning, short-lived nuclei (e.g. ${ }^{13} \mathrm{~N},{ }^{14} \mathrm{O},{ }^{15} \mathrm{O}$, or ${ }^{17} \mathrm{~F}$ with half-life times of 597,71 , 122 , and $65 \mathrm{~s}$, respectively) are produced, which undergo $\beta^{+}$. decay, and work as an energy source for the expansion of the outer, low-density shell (e.g. Starrfield et al. 1972; José et al. 2006). For gamma-ray observations, mainly two species are important as they emit the strongest mono-energetic gamma-ray lines, ${ }^{7} \mathrm{Be}$ and ${ }^{22} \mathrm{Na}$. In addition, ${ }^{13} \mathrm{~N}$ and ${ }^{18} \mathrm{~F}$, from the family of short-lived $\beta^{+}$-unstable nuclei, may be observable due to positron annihilation as a $511 \mathrm{keV}$ gamma-ray flash (see below).

The isotope ${ }^{7} \mathrm{Be}$ is thought to be produced in such a nova explosion via the reaction ${ }^{3} \mathrm{He}(\alpha, \gamma){ }^{7} \mathrm{Be}$. In accelerator experiments, ${ }^{7} \mathrm{Be}$ is produced predominantly in the ground state, $1 / 2^{-}$. However, about $40 \%$ of the time, it is created in its first excited nuclear state, $3 / 2^{-}$, at $429 \mathrm{keV}$ (Parker \& Kavanagh 1963; di Leva et al. 2009), which is the reason that a $429 \mathrm{keV}$ line could also be expected, if the nova envelope was not opaque at this time ${ }^{2}$. The isotope ${ }^{7} \mathrm{Be}$ decays with a half-life time of $T_{1 / 2}^{7 \mathrm{Be}}=53.12 \mathrm{~d}$ (characteristic time of $\tau^{7 \mathrm{Be}}=T_{1 / 2}^{7 \mathrm{Be}} / \ln (2)=$ $76.64 \mathrm{~d}$ ) via electron capture to ${ }^{7} \mathrm{Li}$. This daughter nucleus deexcites to its ground state after 73 fs via the emission of a gamma ray at $E_{0}^{7 \mathrm{Be}}=477.60 \mathrm{keV}$ with a branching ratio of $p^{7 \mathrm{Be}}=10.52 \%$ (Firestone 2003). Depending on the nova model assumptions, the yield of ${ }^{7} \mathrm{Be}$ in $\mathrm{CO}$ novae ranges from $10^{-11}$ to several $10^{-9} M_{\odot}$ (e.g. Hernanz et al. 1996). In general, there are two main types of classical novae, CO- and ONe-types. On the one hand, this classification is based on the final composition of the white dwarf, reflected by the mass and hence the expected burning stages that the progenitor star underwent. Here, a CO white dwarf comes from a star only having burnt $\mathrm{H}$ and $\mathrm{He}$, whereas an $\mathrm{ONe}$ white dwarf progenitor also started $\mathrm{C}$ burning. In return, it is also a plausible assumption, for example in nova model simulations, that white dwarfs below $\sim 1.1 M_{\odot}$ are CO-rich, while more massive ones are of ONe type. This assumption is also based on observational properties of novae, pointing to exactly this white-dwarf composition, for example by measuring emission or absorption lines (see above). In a CO-type nova, the peak temperature allows nuclear burning up to oxygen, with only traces of heavier nuclei. In addition, because ONe white dwarfs have heavier seed nuclei, such as ${ }^{20} \mathrm{Ne}$ or ${ }^{24} \mathrm{Mg}$, in their chemical composition, ONe novae may reach temperatures that can also produce silicon or argon. In the latter case, large amounts of ${ }^{22} \mathrm{Ne}$ are expected to be produced via the reaction chains ${ }^{20} \mathrm{Ne}(p, \gamma)^{21} \mathrm{Na}(p, \gamma)^{22} \mathrm{Mg}\left(\beta^{+}\right)^{22} \mathrm{Na}$ or ${ }^{20} \mathrm{Ne}(p, \gamma)^{21} \mathrm{Na}\left(\beta^{+}\right)^{21} \mathrm{Ne}(p, \gamma)^{22} \mathrm{Na}$, and the subsequent decay $\left(T_{1 / 2}^{22 \mathrm{Na}}=2.6 \mathrm{yr}\right)$ to an excited state of ${ }^{22} \mathrm{Ne}$. The isotope ${ }^{22} \mathrm{Ne}$ then de-excites by the emission of an $E_{0}^{22 \mathrm{Na}}=1274.53 \mathrm{keV}$ gamma ray for $p^{22 \mathrm{Na}}=99.96 \%$ of the time (Firestone 2003). Although this is not expected for CO novae (Hernanz 2014), we perform a search for ${ }^{22} \mathrm{Na}$ in V5668 (see Sect. 2.1).

Theoretical studies (e.g. Clayton \& Hoyle 1974; Leising \& Clayton 1987; José et al. 2001, 2003, 2006; Hernanz \& José 2006; Hernanz 2014) predict a gamma-ray flash around one week before the optical maximum due to short-lived isotopes, such as ${ }^{13} \mathrm{~N}$ and ${ }^{18} \mathrm{~F}$, which decay via positron emission. The true time lag between the initial explosive burning, which results in

\footnotetext{
2 This is true for many other prompt gamma-ray lines which are produced during explosive burning. Here, the $429 \mathrm{keV}$ line serves as a proxy for similar $(p, \gamma)$ reactions of the $\mathrm{CNO}$ cycle, operating in shells of temperatures of $\approx 10^{8} \mathrm{~K}$, which are expected to be opaque at this time.
}

$\lesssim \mathrm{MeV}$ gamma-ray emission, and the optical maximum is fundamentally unknown. Initially, when the nova envelope starts to expand, it is optically thick, so that low-energy photons could not escape. Depending on the nova model set-up, the lag is determined by the time of the maximum temperature (as provided by theory), and the largest extent of the photosphere (optical maximum), resulting in a temporal offset between a few days and two weeks. Throughout this paper, we use a canonical value of the onset of explosive burning of $T_{0}-7 \mathrm{~d}=$ MJD 57095.67 when estimating line fluxes, and release this constraint when searching for the gamma-ray flash (cf. Sects. 2.1 and 2.2). The produced positrons may annihilate quickly and may produce a strong gamma-ray line at $511 \mathrm{keV}$, and a hard X-ray/soft gammaray continuum up to $511 \mathrm{keV}$. For a nova distance of $1 \mathrm{kpc}$, the peak flux in the $75-511 \mathrm{keV}$ band, including the annihilation line, may be as high as $0.2 \mathrm{ph} \mathrm{cm}^{-2} \mathrm{~s}^{-1}$ (e.g. Hernanz 2014). Thus, independent of the direction of a nova with respect to INTEGRAL, this may be seen up to distances of several kpc (Jean et al. 1999). After the peak, the flux declines sharply, and the flash may only be seen by chance.

The ESA gamma-ray observing satellite INTEGRAL (Winkler et al. 2003) was pointed to the galactic centre before and after the optical maximum of V5668. Due to the large field of view of the SPectrometer on INTEGRAL (SPI; Vedrenne et al. 2003) (field of view: $16^{\circ} \times 16^{\circ}$; angular resolution: $2.7^{\circ}$ ), the nova was also observed, as part of other regular observations. SPI measures X-ray and gamma-ray photons in the energy range between 20 and $8000 \mathrm{keV}$, using high-purity Ge detectors. The spectral resolution at $478 \mathrm{keV}$ is $2.1 \mathrm{keV}$ (FWHM). INTEGRAL revolutions 1514,1517 , and 1519 correspond to days -18 to -16 , -11 to -9 , and -5 to -3 with respect to the optical maximum of V5668. After the optical maximum, data from INTEGRAL revolutions 1521 to 1534 , with several observation gaps, allow for high spectroscopic resolution gamma-ray measurements with SPI until day 37, see Fig. 1. The intermediate gaps can be studied in their global gamma-ray emission by the anticoincidence shield (ACS) of SPI, made of 91 scintillating BGO crystals, sensitive to photon (and particle) energies above $\approx 75 \mathrm{keV}$, but without spectral information. The ACS has an almost omni-directional field of view with a rather modest angular resolution $\left(\approx 60^{\circ}\right)$ and high timing capabilities $(\Delta T=50 \mathrm{~ms})$. This allowed us to search for flash-like events during the expected periods around day 7 before the optical maximum. The use of the ACS to detect hard $\mathrm{X}$-ray/soft gamma-ray emission from novae was investigated by Jean et al. (1999).

In this paper, we report a search for gamma-ray line emission from nucleosynthesis ejecta of V5668 using INTEGRAL/SPI, as well as a search for burst-like gamma-ray emission from shortlived nuclei during explosive burning weeks before the optical maximum. In Sect. 2, we describe the analyses of SPI and its ACS data, exploiting spectral, temporal, and directional information, and derive physical parameters. The implications of the analysis results are discussed in Sect. 3.

\section{Data analysis}

\subsection{SPI analysis}

We analyse the spectral and the temporal domain for the expected gamma-ray lines at 429, 478, 511, and $1275 \mathrm{keV}$. Starting at $T_{1}=T_{0}-7 \mathrm{~d}=$ MJD 57095.67, the total exposure in our SPI data set until day $+37\left(T_{2}=T_{0}+37 \mathrm{~d}=\right.$ MJD 57139.67) is $T_{\exp }=1.53 \mathrm{Ms}<\Delta T=T_{2}-T_{1}=44 \mathrm{~d} \approx 3.80 \mathrm{Ms}$. This is reduced further by observational gaps in the regular INTEGRAL 


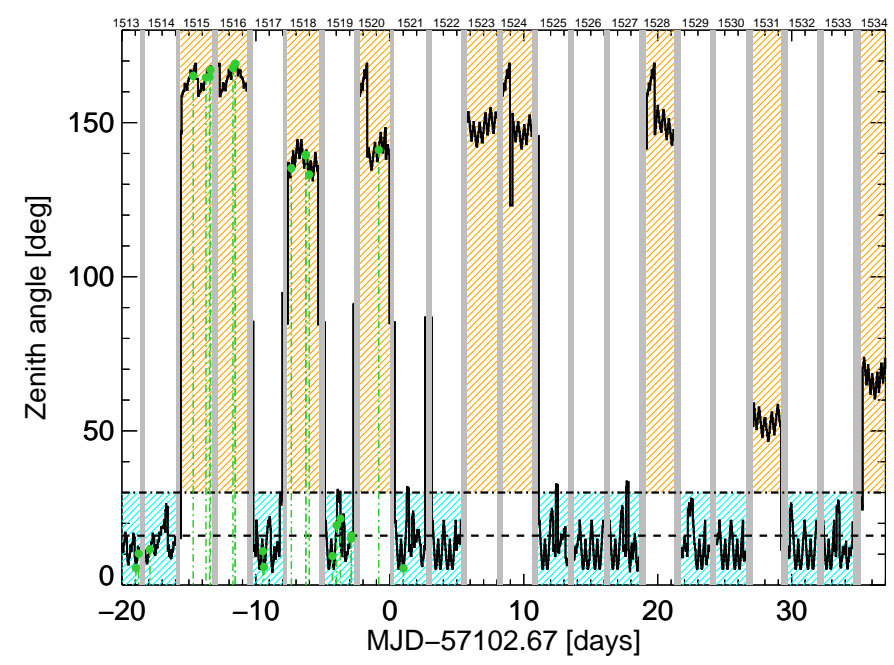

Fig. 1. Position of V5668 Sgr (zenith angle, black data points) with respect to the SPI on-axis frame as a function of time in days from the nova optical maximum (MJD 57102.67). INTEGRAL revolution numbers are at the top. The black dashed and dot-dashed horizontal lines correspond to the SPI fully coded $\left(16^{\circ}\right)$ and partially coded $\left(30^{\circ}\right)$ field of view, respectively. The hatched cyan areas mark the times used for SPI on-axis analysis, whereas during the times marked by the orange hatched areas, off-axis analysis was performed using the ACS (see Sect. 2.3.2). The grey regions show the perigee passages of INTEGRAL, i.e. when no data is taken. The green dots indicate the times when the SPI-ACS search for flash-like events found its candidates (see Sect. 2.2; all aspect angles have been used for this search, but only from day -20 to day +3 ).

observation program, data selection criteria, such as orbit phases (0.1-0.9 to avoid the Earth's radiation belts) and onboard radiation monitor rates acceptance windows (to avoid charged particle showers and solar flares), and detector dead time. The total observation time is $T_{\mathrm{obs}}=1.06 \mathrm{Ms}$. SPI data are dominated by background from cosmic-ray bombardment of satellite and instruments, leading to decay and de-excitation photons.

The spectrometer data are analysed by a maximum likelihood method, comparing measured Ge detector data to models of celestial emission and background. We use a self-consistent, high spectral resolution, background modelling procedure (e.g. Diehl et al. 2014; Siegert et al. 2016; Siegert 2017) to extract spectra in the energy ranges 70-530 keV and 1240-1310 keV. In general, the modelled time patterns (count sequences in different detectors per unit time) for each model component are fitted to the measured time pattern of the data by minimising the Cash statistic (Cash 1979):

$C\left(D \mid \theta_{i}\right)=2 \sum_{k}\left[m_{k}-d_{k} \ln m_{k}\right]$,

which accounts for Poisson-distributed photon count statistics. In Eq. (1), $d_{k}$ are the measured and $m_{k}$ the modelled data, which are matched (fitted) to $d_{k}$ by adjusting the intensity scaling parameters $\theta_{(t)}$ of the model components, which are possibly time-dependent:

$m_{k}=\sum_{t_{S}} \sum_{j} R_{j k} \sum_{i=1}^{N_{S}} \theta_{i, t_{S}} M_{i j}+\sum_{t_{B}} \sum_{i=N_{S}+1}^{N_{S}+N_{B}} \theta_{i, t_{B}} B_{i k}$.

Here, we describe the model in each half-keV energy bin $k$ as a superposition of $N_{S}$ celestial models $M_{i j}$, to which the instrumental imaging response (coded-mask response, $R_{j k}$ ) is applied for each image element $j$, and $N_{B}$ background models $B_{i k}$. For the background model, we use the information gathered over the INTEGRAL mission years, separating long-term stable or smoothly varying properties, such as detector degradation (linear within half a year) or solar activity (solar cycle anti-correlates with cosmic-ray intensity rate), from shortterm variations, such as solar flares and general pointing-topointing variations. Instrumental gamma-ray continuum and line backgrounds are treated separately, according to their different physical origins inside the satellite. Each instrumental line imprints a certain pattern onto the gamma-ray detector array, depending on the distribution of the radiating material inside the satellite. These patterns are constant over time because the material distribution does not change. Only detector failures lead to a change in those patterns as double scattering photons in dead detectors are then seen as single events in working neighbouring detectors. Different activation rates (cosmic-ray bombardment) and isotope decay times then lead to different amplitudes in those patterns, which are determined using Eqs. (1) and (2). For any specific process, these patterns are constant in time; however, for single energy bins (typically $0.5 \mathrm{keV}$, cf. instrumental resolution of 2-3 keV), these patterns may change due to different degradation strengths in the 19 detectors. Hence, we determine the detector patterns by performing a spectral decomposition (statistical fit) in each of the detectors on a three-day timescale (viz. one INTEGRAL orbit). This allows us to trace the degradation and the general response properties of all detectors with time, and at the same time smears out celestial contributions, because the varying time-patterns of the coded-mask response in combination with the INTEGRAL dithering strategy average out. The procedure and functions to determine the spectral response parameters have already been discussed in Siegert et al. (2016) and Siegert (2017); see also Diehl et al. (2018) for an analysis of the 15-yr SPI data base.

By performing this maximum likelihood estimation for each of the spectral bins in the energy region of interest, we create spectra for each source or general emission morphology. In the case of V5668, the only $\left(N_{S}=1\right)$ celestial model is a pointsource at the position of the nova, $M_{1 j}=\theta_{1, t_{S}} \delta\left(l-l_{0}\right) \delta\left(b-b_{0}\right)$. The decay time of ${ }^{7} \mathrm{Be}$ of 77 days, and the possible gamma-ray flash before the optical maximum leads to two analysis cases: (1) integration over the entire exposure time to obtain a maximum of sensitivity for the longer-lived nucleosynthesis products in fine energy resolution (no time-dependence, $\theta_{1, t_{S}} \rightarrow \theta_{1}$ ) and (2) several time intervals of $2-3 \mathrm{~h}$ to study the transient behaviour in broader energy binning to enhance the sensitivity of observing a gamma-ray flash, and also to trace the radioactive decay of ${ }^{7} \mathrm{Be}$ (light curve).

In Figs. 2a and b, the average spectra for V5668 between days -7 and 37 after the optical maximum are shown. In both energy bands, no significant excess is seen, and the spectra are consistent with zero flux. We derive upper limits on the fluxes by assuming an average line shift of $-1000 \mathrm{~km} \mathrm{~s}^{-1}$ (Molaro et al. 2016; Tajitsu et al. 2016), which corresponds to shifted gamma-ray lines centroids of $479.1 \mathrm{keV}$ and $1278.8 \mathrm{keV}$, respectively. The line broadening is adopted as $8 \mathrm{keV}$ for the $478 \mathrm{keV}$ line, and $21 \mathrm{keV}$ for the $1275 \mathrm{keV}$ line (FWHM, e.g. Hernanz 2014; Siegert 2017 instrumental resolution $2.08 \mathrm{keV}$ at $478 \mathrm{keV}$, and $2.69 \mathrm{keV}$ at $1275 \mathrm{keV})$. At days $T_{0}+(15 \pm 22)$, the $3 \sigma$ upper limit on the $478 \mathrm{keV}$ line flux is estimated to be $8.2 \times 10^{-5} \mathrm{ph} \mathrm{cm}^{-2} \mathrm{~s}^{-1}$. The $3 \sigma$ upper limit on the $1275 \mathrm{keV}$ line flux is $7.6 \times 10^{-5} \mathrm{ph} \mathrm{cm}^{-2} \mathrm{~s}^{-1}$. 


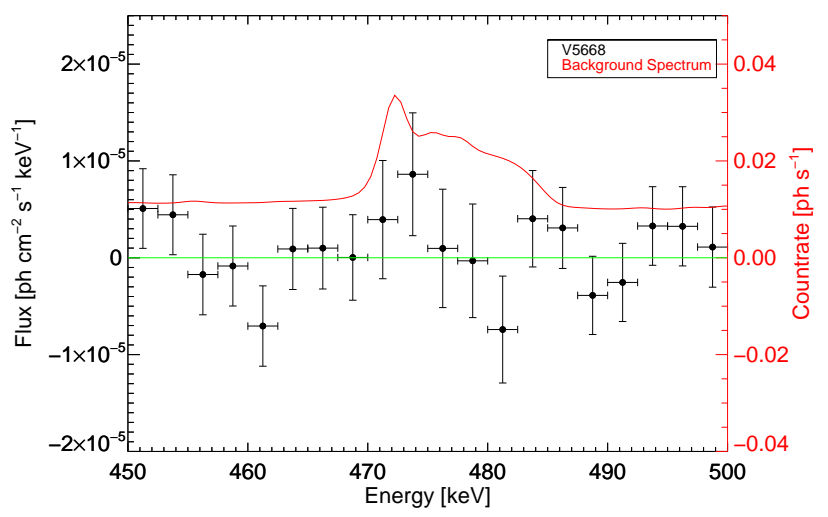

(a) ${ }^{7} \mathrm{Be} 478 \mathrm{keV}$ line region.

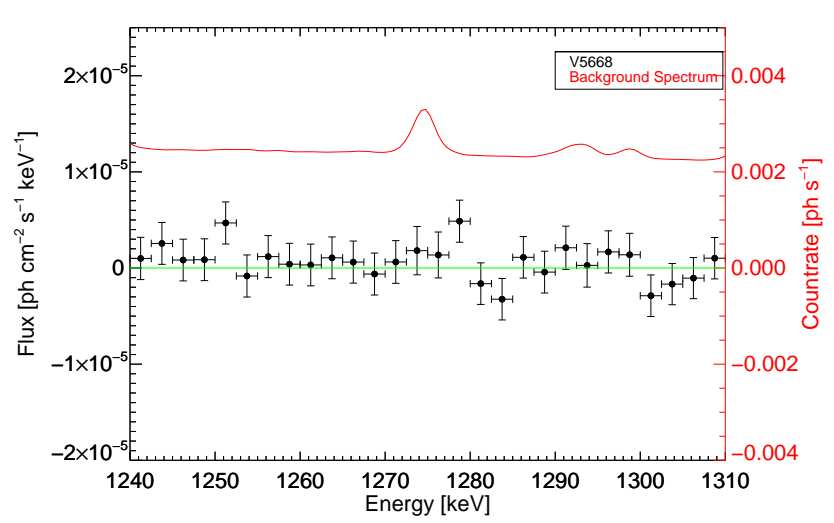

(b) ${ }^{22} \mathrm{Na} 1275 \mathrm{keV}$ line region.

Fig. 2. Spectra (black data points, left-hand axes, $2.5 \mathrm{keV}$ binning) for V5668, integrated between days -7 and +37 after the optical maximum. Also shown are the SPI background spectra (red, right-hand axes) in the energy range 450-500 keV (panel a) and 1240-1310 keV (panel $b$ ). The background is well suppressed, and no significant linelike or continuum excesses above background can be identified. The zero-flux level is indicated by green solid lines. See text for details.

In order to derive an upper limit on the mass of ${ }^{7} \mathrm{Be}$, the expected flux of the $478 \mathrm{keV}$ line from the radioactive decay law,

$F^{7 \mathrm{Be}}(t)=\frac{M^{7 \mathrm{Be}} p^{7 \mathrm{Be}}}{4 \pi d^{2} N^{7 \mathrm{Be}} u \tau^{7 \mathrm{Be}}} \exp \left(-\frac{t-\Delta t}{\tau^{7 \mathrm{Be}}}\right)$

is used. In Eq. (3), $M^{7 \mathrm{Be}}$ is the synthesised mass of ${ }^{7} \mathrm{Be}$ seen to decay, $d$ is the distance to $\mathrm{V} 5668, N^{7 \mathrm{Be}}=7$ is the number of nucleons in ${ }^{7} \mathrm{Be}, u=1.66 \times 10^{-27} \mathrm{~kg}$ is the atomic mass unit, $\Delta t$ is fixed to 7 days before the optical maximum, and $p^{7 \mathrm{Be}}=10.52 \%$ is the probability of emitting a $478 \mathrm{keV}$ photon after the decay. The flux limits convert to a ${ }^{7} \mathrm{Be}$ mass limit of $M_{3 \sigma}^{7 \mathrm{Be}}<4.8 \times 10^{-9}\left(\mathrm{~d} k p c^{-1}\right)^{2} M_{\odot}$, and a ${ }^{22} \mathrm{Na}$ mass limit of $M_{3 \sigma}^{22 \mathrm{Na}}<2.4 \times 10^{-8}\left(\mathrm{~d} k p c^{-1}\right)^{2} M_{\odot}$. Using the distance values from Banerjee et al. (2016) or Jack et al. (2017) of $\approx 1.6 \mathrm{kpc}$, the limits on the ejected masses yield $M_{3 \sigma}^{7 \mathrm{Be}}<1.2 \times 10^{-8} M_{\odot}$, and $M_{3 \sigma}^{22 \mathrm{Na}}<6.1 \times 10^{-8} M_{\odot}$, respectively. Molaro et al. (2016) estimated a ${ }^{7} \mathrm{Be}$ mass of $7 \times 10^{-9} M_{\odot}$ from their UV spectra. This is consistent with our limit. Assuming their amount of ejected mass, the non-detection by INTEGRAL/SPI requires the distance to the nova V5668 to be larger than $1.2 \mathrm{kpc}$ ( $3 \sigma$ lower limit).

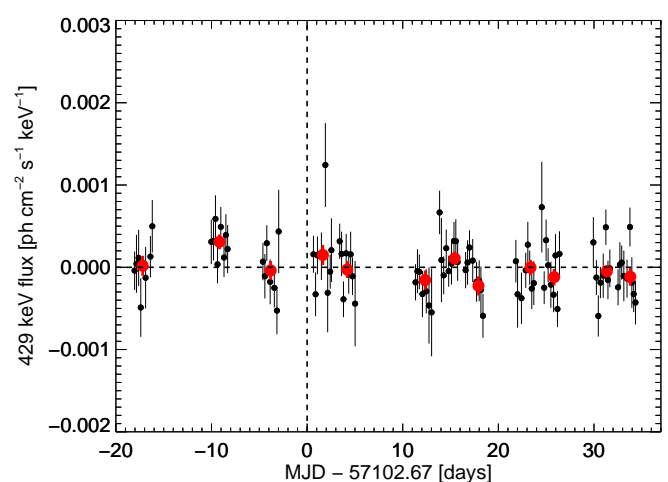

(a) $429 \mathrm{keV}$ line.

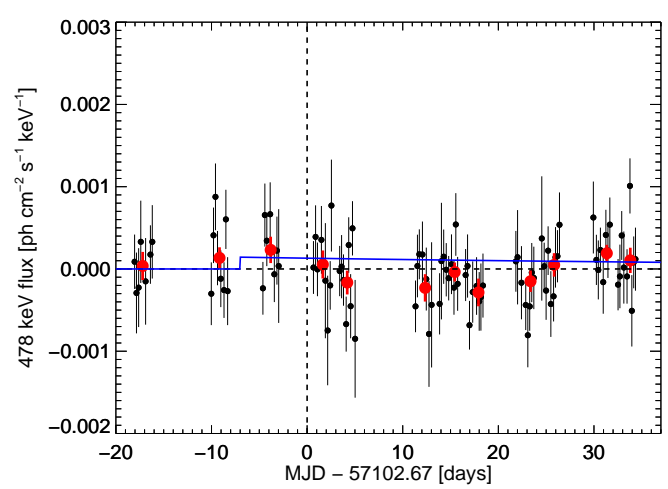

(b) $478 \mathrm{keV}$ line.

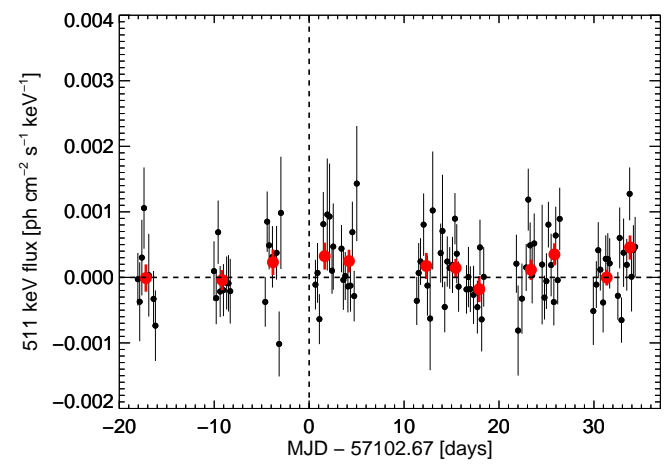

(c) $511 \mathrm{keV}$ line.

Fig. 3. Light curves of the gamma-ray lines at $429 \mathrm{keV}$ $\left({ }^{3} \mathrm{He}(\alpha, \gamma){ }^{7} \mathrm{Be}^{*}\right.$, panel $\left.a\right), 478 \mathrm{keV}\left({ }^{7} \mathrm{Be}(E C)^{7} \mathrm{Li}\right.$, panel $\left.b\right)$, and $511 \mathrm{keV}$ $\left(\mathrm{e}^{+}+\mathrm{e}^{-} \rightarrow \gamma+\gamma\right.$, panel $\left.c\right)$. Shown are measured flux values in the energy bands $425-433 \mathrm{keV}, 475-483 \mathrm{keV}$, and 506-516 keV, respectively, for $2-3 \mathrm{~h}$ time bins (black dots), and $\approx 2$-day intervals (red dots), with respect to the optical maximum at $T_{0}=$ MJD 57102.67 (vertical dashed line). The zero flux levels are indicated by a horizontal dashed line. No significant excess $(>3 \sigma)$ is found during the observations. In order to derive an upper limit on the flux from the decay of ${ }^{7} \mathrm{Be}$, an exponential radioactive decay law has been fitted to the data points. As the true time of explosive burning of V5668 is not known, a canonical time offset of $\Delta t=-7 \mathrm{~d}$ is used. The $3 \sigma$ upper limit on this decay law is shown as solid blue line in panel b. An upper limit on the annihilation flux at that time is not possible as INTEGRAL was pointed away from the source. See text for more details.

In Fig. 3a-c, the gamma-ray light curves of the 429, 478, and $511 \mathrm{keV}$ lines are shown, respectively. There is no significant excess in the gamma-ray light curves from ${ }^{7} \mathrm{Be}$ or positron annihilation from the position of V5668. Around day -10 , the mean $429 \mathrm{keV}$ line flux has a significance of $3.6 \sigma$ above 
the background. However, in the continuum band between 70 and $520 \mathrm{keV}$, no signal is detected $\left(<0.018 \mathrm{ph} \mathrm{cm}^{-2} \mathrm{~s}^{-1}\right)$, and we consider this to be a statistical fluctuation. During days -8 to -6 , for example, INTEGRAL observed other parts of the sky, and V5668 was not in the field of view (see angular position of V5668 with respect to the SPI on-axis frame in Fig. 1). Either the source was in the field of view of SPI and IBIS or the backside of the veto-shields are exposed to the source. Fitting the radioactive decay, Eq. (3), to the data in Fig. 3b obtains an upper limit on the synthesised ${ }^{7} \mathrm{Be}$ mass of $M_{3 \sigma}^{7 \mathrm{Be}}<6.4 \times 10^{-9}\left(\mathrm{dkpc}^{-1}\right)^{2} M_{\odot}$. Using the $1.6 \mathrm{kpc}$ distance estimate as before, the mass is constrained to $M_{3 \sigma}^{7 \mathrm{Be}}<1.6 \times 10^{-8} M_{\odot}$. Assuming again the mass estimate by Molaro et al. (2016), V5668 must be further away than $d_{3 \sigma}^{7 \mathrm{Be}}>1.1 \mathrm{kpc}$. These limits become more constraining if the time-dependence of the expected signal (radioactive decay) is taken into account, i.e. $M_{3 \sigma}^{7 \mathrm{Be}}<4.8 \times 10^{-9}\left(\mathrm{dkpc}^{-1}\right)^{2} M_{\odot}$ and $d_{3 \sigma}^{7 \mathrm{Be}}>1.2 \mathrm{kpc}$.

\subsection{ACS analysis}

We used the SPI-ACS to search for burst-like emission during the three weeks before the optical maximum of the nova. SPI was not pointed in the direction of V5668 for most of this time, and the ACS has an omni-directional field of view to possibly detect such a feature. The nova model light curve by Hernanz (2014) provides a template for the temporal evolution of the expected gamma-ray flash. Between 75 and $511 \mathrm{keV}$, the light curve rises with a maximum at hour 1 , sharply decreases exponentially to hour 4, and then fades away. We interpolated this coarsely sampled model onto our 1 min time binning of the (total) ACS rate, $R_{\mathrm{ACS}}^{\text {tot }}(t)$. In particular, we performed a search of excess signals in the ACS rate, i.e. nova flash candidate events, using a maximum likelihood method. This requires a background model for the ACS counts at any time $B(t)$, an intensity scaling parameter $a_{0}$ for the flash model $F(t)$, and a temporal variable $t_{0}$ that determines the flash time. During the three weeks before the optical maximum, we tested a grid of 200 amplitudes (source intensities), equally spaced between 0.0 and 0.2 of the peak amplitude of $0.21 \mathrm{ph} \mathrm{cm}^{-2} \mathrm{~s}^{-1}$, and used 400 time bins, equally spaced inside each INTEGRAL revolution, i.e. approximately $7.5 \mathrm{~min}$ steps. Due to irregular particle events when entering and exiting the radiation belts, we limited the search to times when the ACS rate shows a smooth, non-erratic behaviour. This typically cut out a few hours after the belt exits. We analysed INTEGRAL revolutions 1513 to 1521 , i.e. days -20 to +3 in this way. The total model, $M\left(t ; a_{0}, t_{0}\right)$, that is tested on the specified grid through the $\mathrm{ACS}$ rate in each orbit is then

$M\left(t ; a_{0}, t_{0}\right)=B(t)+a_{0} \times F\left(t-t_{0}\right)$.

As a background model for the ACS, we use a median filter of one hour applied to the ACS rate itself, $B(t)=$ $\operatorname{median}\left(R_{\mathrm{ACS}}^{\mathrm{tot}}(t), 60 \mathrm{~min}\right)$. This smears out features on this and shorter timescales, which may then be captured by our nova model light curve.

In each revolution, we find several candidates by iteratively accepting the next to maximum likelihood value in the marginalised probability density function of $t_{0}$. Table 1 summarises the nova flash candidate events with a statistical significance of more than $3.9 \sigma\left(p<10^{-4}\right)$. In the following, we perform additional analyses to possibly distinguish between solar events (particle and photon), gamma-ray bursts (GRBs), the nova itself, or other X-ray transients.

\subsection{Distinguishing between event candidates}

\subsubsection{Temporal characteristics}

The temporal characteristic of a candidate is a first indicator of its origin. While all candidates have been identified by using a nova model light curve, any emission above the background captured by this short-duration model will improve the fit, but is not necessarily attributable to a nova. In general, a sharp rise and fast decay feature might point to a nova origin. However, solar flare events also show this behaviour, but in this case it is followed by an irregular and strong particle flux which is also measured by the ACS. Very strong GRBs on timescales of seconds to minutes will also be captured by our method, but can easily be identified as such by investigating the residuals of the fit as their temporal profiles are more stochastic than our smooth nova model. We provide the duration of the candidate event, $\Delta T$, as well as the $\chi^{2}$ goodness-of-fit value of the nova model in Table 1, and mark possible GRBs and non-nova-like events in the comments column.

\subsubsection{Directional information}

The ACS consists of 91 individual BGO blocks, arranged in a hexagonal structure surrounding SPI up to its mask. If an event originates from a particular direction (point source), the facing side of the ACS will record more counts than the averted side. This can be expressed by an anisotropy factor of the different ACS subunits, which consist of rings at different positions with respect to the SPI camera, and with different BGO thicknesses, ranging from $16 \mathrm{~mm}$ at the top (upper collimator ring, UCR1) to $50 \mathrm{~mm}$ at the lower veto shield directly below SPI. Most of the subunits alone are not suitable to perform such an anisotropy analysis because they are partially shadowed by IBIS, which is the other main instrument on INTEGRAL (Ubertini et al. 2003). On the level of the Ge detector array, the path between two opposing BGO crystals is blocked by the camera itself, so that the information may be skewed. By performing a similar analysis for a major solar flare, Gros et al. (2004) concluded that the UCR 1 is the most sensitive subunit to infer coarse directional information. Gros et al. (2004) defined the anisotropy parameter as $A=(R-L) /(R+L)$, where $R$ and $L$ are the rates of opposing UCR 1 hemispheres, i.e. the total count rates of each of the three BGO detectors. This allowed the authors to identify azimuthal directions if the source direction (aspect) is perpendicular to the crystal.

For general incidence angles to the veto shields, this anisotropy smears out, and the omni-directional INTEGRAL response is required (see e.g. Savchenko et al. 2017a,b, for the search of electromagnetic counterparts of the gravitational wave). We perform an analysis of the INTEGRAL veto-shields and ISGRI count rates. Depending on the source position (Fig. 1) and the expected spectrum, the count rates vary accordingly. Comparing the ACS-to-Veto count ratio, and the ACS-to-ISGRI count ratio with the prediction from the source spectrum, a localisation is possible for strong sources like GRBs. For the weak candidate events, we use this response to distinguish between a possible nova or another origin. In Fig. 4, the top three panels show the IBIS/ISGRI, IBIS/Veto, and SPI-ACS count rates. The bottom panel illustrates the expected count ratios from the direction of V5668 with estimated uncertainties (orange and blue bands), together with the actual measured count ratios for candidate events for which ISGRI data is available (cf. Fig. 1, perigee passages). The spectral properties during the luminosity peak of the gamma-ray transient have been assumed to follow a 
T. Siegert et al.: Gamma-ray observations of Nova Sgr 2015 No. 2 with INTEGRAL

Table 1. Nova candidate events, identified using the SPI-ACS rate and the nova model light curve by Hernanz (2014).

\begin{tabular}{cccrrrrcc}
\hline \hline$t-T_{0}$ & $T_{1}$ & $T_{2}$ & $\Delta T$ & $a_{0}$ & $\chi^{2} / v$ & $\Delta \mathcal{L}[\sigma]$ & $H R_{500}[\sigma]$ & Comments \\
\hline-455.2 & 83.713 & 83.715 & 181.3 & $>0.2$ & 234.6 & $>1000$ & -0.0 & GRB \\
-450.2 & 83.914 & 83.922 & 690.8 & $0.092(7)$ & 8.0 & 13.1 & +0.0 & not nova-like \\
-429.8 & 84.762 & 84.770 & 690.8 & $0.065(7)$ & 3.2 & 9.3 & -0.4 & too early \\
-352.5 & 87.993 & 87.994 & 25.7 & $0.049(8)$ & 22.3 & 6.1 & +2.2 & GRB \\
-329.7 & 88.943 & 88.950 & 604.5 & $0.050(7)$ & 3.3 & 7.1 & +2.9 & Sun/GOES \\
-322.0 & 89.205 & 89.223 & 1555.0 & $0.030(8)$ & 1.2 & 3.9 & +2.7 & Sun/GOES \\
-321.7 & 89.278 & 89.285 & 605.1 & $0.065(7)$ & 8.2 & 9.3 & -0.8 & not nova-like \\
-281.2 & 90.959 & 90.960 & 69.2 & $0.183(8)$ & 60.4 & 22.9 & +1.3 & GRB \\
-277.6 & 91.105 & 91.138 & 2851.0 & $0.054(7)$ & 85.9 & 7.7 & +3.0 & Sun/GOES \\
-277.0 & 91.140 & 91.142 & 129.2 & $0.155(7)$ & 120.0 & 22.1 & +1.5 & GRB \\
\hline$-227.4^{c}$ & 93.197 & 93.200 & 259.1 & $0.054(7)$ & 4.7 & 7.7 & +0.7 & not nova-like \\
$-225.5^{c}$ & 93.260 & 93.305 & 3887.8 & $0.031(8)$ & 1.9 & 3.9 & +0.6 & GOES \\
$-176.7^{c}$ & 95.300 & 95.340 & 3455.4 & $0.064(7)$ & 3.2 & 9.1 & +0.5 & false response \\
$-150.8^{a}$ & 96.395 & 96.400 & 432.4 & $<0.054$ & 4.5 & $<7.7$ & +0.3 & weak \\
$-150.8^{a}$ & 96.402 & 96.403 & 95.6 & $<0.054$ & 4.5 & $<7.7$ & +0.7 & GRB \\
$-144.5^{c}$ & 96.650 & 96.661 & 950.5 & $0.036(8)$ & 1.9 & 4.5 & -0.0 & false response \\
$-103.3^{c}$ & 98.362 & 98.405 & 3715.1 & $0.048(7)$ & 1.8 & 6.9 & +0.7 & false response \\
-95.6 & 98.687 & 98.714 & 2332.8 & $0.037(7)$ & 1.8 & 5.3 & +3.6 & Sun \\
$-88.8^{c}$ & 98.972 & 98.999 & 2332.8 & $0.034(7)$ & 2.2 & 4.9 & -1.7 & GOES \\
$-69.1^{a}$ & 99.781 & 99.782 & 86.4 & $>0.2$ & 18.2 & $>1000$ & -1.2 & GRB/GOES \\
$-69.1^{a}$ & 99.793 & 99.799 & 518.8 & $<0.069$ & 20.4 & $<8.0$ & +3.7 & Sun/weak \\
\hline-20.3 & 101.825 & 101.866 & 3542.4 & $0.034(7)$ & 1.5 & 4.9 & +1.0 & too late \\
+24.3 & 103.686 & 103.699 & 1123.2 & $0.049(7)$ & 2.6 & 7.0 & -0.0 & after V-maximum \\
\hline & & & & & & & &
\end{tabular}

Notes. The columns contain the following values: $t-T_{0}$ shows the temporal difference between the optical maximum $T_{0}$ and the event time in units of hours; $T_{1}$ and $T_{2}$ are the start and end times of the feature in units of MJD+57 000; $\Delta T$ is the duration in units of s; $a_{0}$ is the fitted amplitude of the nova model; $\chi^{2} / v$ is the goodness-of-fit value, weighted by the number of degrees of freedom (reduced $\chi^{2}$ ), indicating whether a nova model is appropriate to express the light curve $; \Delta \mathcal{L}$ is the significance of the feature over the background in units of $\sigma$; and $H R_{500}$ is the hardness ratio difference, comparing the times before, during, and after the event in units of $\sigma$. The last column provides plausible source identifications, based on the other columns, coincidences with GOES X-ray data, or the INTEGRAL response. The horizonal dashed lines indicate times for which the gamma-ray flash is "most probably" expected to occur (Gomez-Gomar et al. 1998), i.e. between days 2 and 10 before the visual maximum.

${ }^{(a)}$ Two events which are coincident within a few minutes are captured by the search method as only one. This happens for example when a strong GRB precedes a solar particle event. Here, upper limits are given for fluxes and significances. ${ }^{(b)}$ Because the nova light curve model is not parametrised and thus is very stiff, the resulting $\chi^{2}$ values are subject to extreme values and variations. We take this into account by also considering apparently bad $\chi^{2}$ values, up to $25 \sigma$ deviations from the optimum. This declares reduced $\chi^{2}$ values below $\approx 4$ as good, given the number of degrees of freedom of around $160 .{ }^{(c)}$ Intriguing events discussed in the main text although formally excluded by specific diagnostics.

power-law with a spectral index of -1 . The angular dependence of the INTEGRAL all-sky response is mostly sensitive to spectral shapes between 50 and $300 \mathrm{keV}$, so that the model approximation remains valid although the spectrum, as estimated by Hernanz (2014), may be more complex ${ }^{3}$. For the purpose of localisation, it is sufficient to adopt a simple power-law spectrum. If the measured points coincide with this expectation, the event origin is likely to be from the direction of the nova, although may not be caused by the nova.

\subsubsection{Spectral hardness}

The distinction between solar events and X-ray transients can be augmented further by investigating the count rate in SPI during the time of a candidate event. While transient X-ray sources predominantly emit up to $500 \mathrm{keV}$ photon energies, often with an exponential cut-off (e.g. Done et al. 2007), solar flares can also show a strong increase in the high-energy continuum up to several $\mathrm{MeV}$, and in addition de-excitation lines from ${ }^{16} \mathrm{O}^{*}$ $(6.129 \mathrm{MeV}),{ }^{12} \mathrm{C}^{*}(4.438 \mathrm{MeV})$, and ${ }^{2} \mathrm{H}^{*}(2.223 \mathrm{MeV})$, for

3 Between 50 and $300 \mathrm{keV}$, the nova model by Hernanz (2014) indeed follows a power law with index -1 . example (Gros et al. 2004; Kiener et al. 2006). We note however, that solar X-class flares which produce high-energy gamma-ray lines appear to be rare events (<10\%, Vestrand et al. 1999), but can be identified as such by SPI and its subsystems. The SPI-ACS transparency is increased with increasing energy, so that we can utilise the information from the entire SPI instrument, i.e. ACS and Ge detectors. The measured residual Ge detector counts that pass the shield are thus a convolution of the ACS-transparency with the source emissivity. We define a hardness ratio between the energy bands 500-8000 keV and $20-500 \mathrm{keV}, H R_{500}=F_{500-8000} / F_{20-500}$, in 1 min steps, to obtain another basis of decision for the nova flash candidates. In general, the hardness ratio $H R_{500}$ is a smoothly varying function with time and is typically around 0.4 in SPI raw data. This value can change over time, for example due to X-ray transients, showing a decreased $H R_{500}$ with respect to the average, whereas solar events increase the ratio. We analyse $H R_{500}$ during each candidate event, and compare the value to the averaged hardness ratio, integrated over one hour before and one hour after the event. We express the change in hardness in units of $\sigma$ in Table 1. Positive deviations would indicate a solar origin, negative ones possibly $\mathrm{X}$-ray transients. 


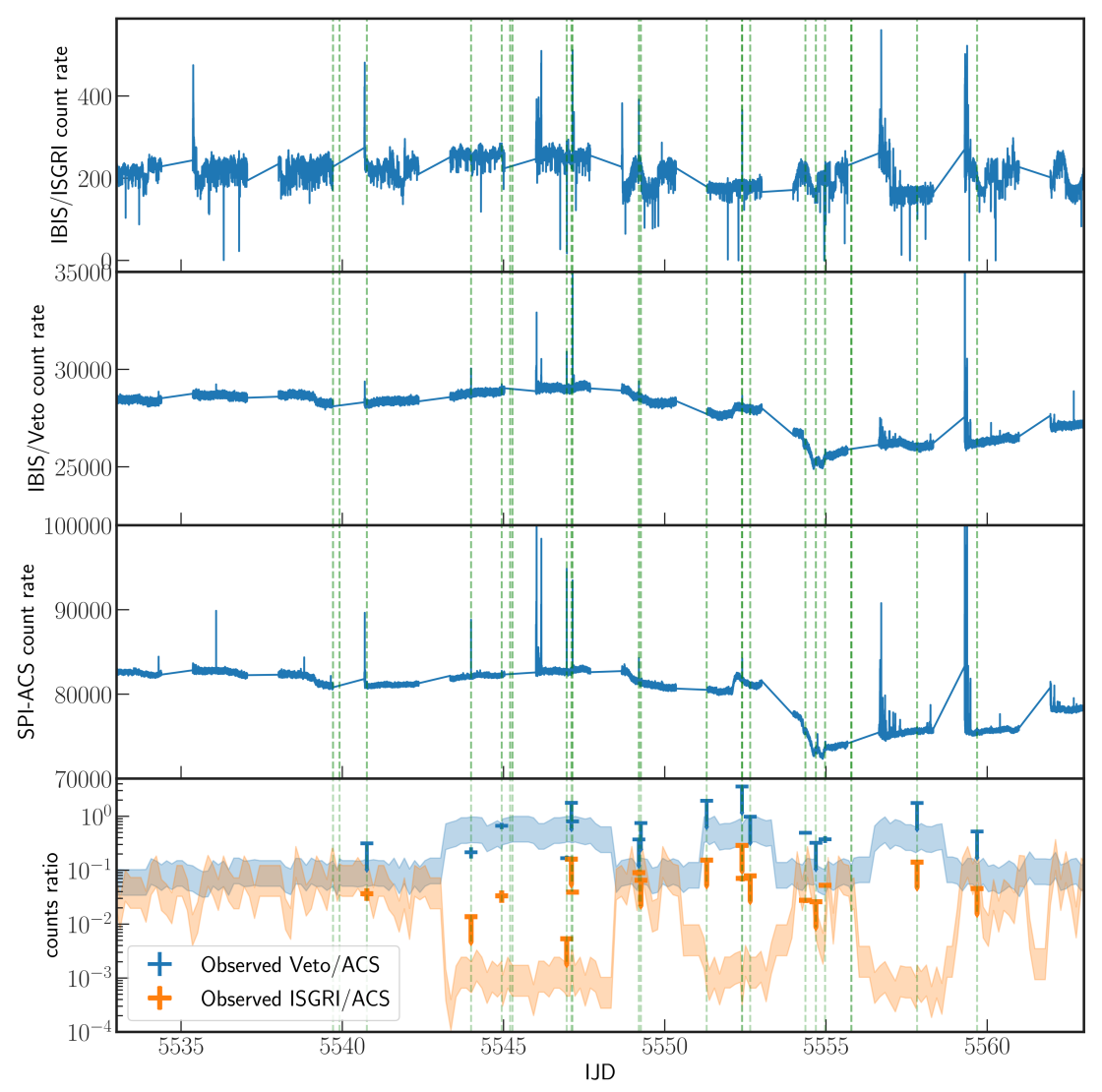

Fig. 4. INTEGRAL response to the nova V5668 Sgr. Top three panels: measured counts rates of different instruments aboard INTEGRAL in $100 \mathrm{~s}$ time bins (blue data points). From top to bottom: IBIS/ISGRI, IBIS/Veto, and SPI-ACS. Bottom panel: count ratios between the Veto and the ACS (blue), and ISGRI and the ACS (orange) if a source at the position of V5668 exposed INTEGRAL with a nova-like spectrum. The blue and red data points mark the actually measured ratios during the 23 event times (vertical dashed green lines). See text for details.

\subsubsection{Event identification and discussion}

Based on the above criteria, we exclude 9 of the 23 candidates because of their temporal fitting residuals (GRBs, not nova-like in general), and 5 events are presumably from the direction of the Sun, based on an increased hardness ratio. Based on theoretical predictions (Gomez-Gomar et al. 1998) of such a nova flash 2 to 10 days before the optical maximum, we consider that one event is too early to originate from $\operatorname{V5668}\left(t-T_{0}=-429.8 \mathrm{~h} \approx-18 \mathrm{~d}\right)$. Two other events may be considered too late, happening close to or even after the $V$-band maximum. Several events coincide closely with GOES X-ray data ${ }^{4}$, even though the hardness ratio is unchanged. Two additional events can be excluded in this way. The remaining three ACS features can be assigned a direction off the nova location and do not show a decreased $H R_{500}$, which could be expected if a transient X-ray source was near.

Even though our decision-making can exclude any of the candidates from being associated with V5668, the case for six of them remains intriguing. This may be either because the response is exactly met but the light curve does not appear nova-like or vice versa, or because an event is only close in time with GOES and not strictly coincident (marked (c) in Table 1). These candidates would either be classified as "fast" $\left(T_{0}-5 \mathrm{~d}\right.$ to $\left.T_{0}-2 \mathrm{~d}\right)$ or "moderately fast" $\left(T_{0}-10 \mathrm{~d}\right.$ to $\left.T_{0}-5 \mathrm{~d}\right)$ novae, based on the gamma-ray flash occurrence time (Gomez-Gomar et al. 1998). The measured peak fluxes are similar for all candidates, ranging between 6.5 and $13.4 \times 10^{-3} \mathrm{ph} \mathrm{cm}^{-2} \mathrm{~s}^{-1}$ in the energy regime of the SPI-ACS. These values are systematically uncertain by about $60 \%$ because

4 See https://satdat.ngdc.noaa.gov/sem/goes/data/new_ plots/2015/goes15/qc/g15_xrs-euv_1m_qc_20150301-00h_ 20150331-24h.pdf for GOES data around the time of the V5668 outburst. the true effective area at each individual event is not known. The statistical uncertainties range between $10 \%$ and 25\%. Taking all uncertainties into account, these events would correspond to luminosities $\approx 4$ to 50 times higher than the model assumption. This is a factor of 2-7 above the inferred values from Banerjee et al. (2016, $1.54 \mathrm{kpc}$ ) or Jack et al. (2017, $1.6 \mathrm{kpc})$. This discrepancy might be due to our use of the nova model light curve, its shape, and its peak amplitude, being uncertain by about an order of magnitude (e.g. Hernanz et al. 1997; Hernanz $2005,2014)$. With increased information of nuclear cross sections and more elaborate modelling approaches the estimates become more realistic, even though large uncertainties can still arise, for example when considering the initial conditions.

The ACS feature with the best-fitting temporal behaviour $\left(\chi^{2} / v=1.8\right)$ occurred 4.3 days $(103.3 \mathrm{~h})$ before the optical maximum, with a significance of $6.9 \sigma$ above the median ACS count rate. Its duration is at least $3700 \mathrm{~s}$, and afterwards it drowns into the background again. The hardness ratio is not significantly increased $(+0.7 \sigma)$, so that a solar or nova origin can neither be excluded nor suggested. The response function excludes that this source, by about $3 \sigma$, comes from the direction of V5668. Based on only the hardness ratio, the feature around day 3.7 (h 88.8) before the optical maximum $\left(\Delta H R_{500}=-\right.$ $1.7 \sigma$ ) would suggest an X-ray source, though with low significance. During these two events, INTEGRAL was pointed towards V5668, and the source was in the partially coded field of view of IBIS and SPI. No hard X-ray/soft gamma-ray emission (70-520 keV) was detected during this time for both instruments (SPI: $<0.017 \mathrm{ph} \mathrm{cm}^{-2} \mathrm{~s}^{-1}$ at day $-4.3,<0.049 \mathrm{ph} \mathrm{cm}^{-2} \mathrm{~s}^{-1}$ at day -3.7 ; IBIS: $<0.003 \mathrm{ph} \mathrm{cm}^{-2} \mathrm{~s}^{-1} ; 3 \sigma$ upper limits). The strongest signal in the ACS rate occurred 7.4 days $(176.6 \mathrm{~h})$ before the visual maximum with $9.1 \sigma$ above the background level. This event was preceded by another, but weaker $(<3.9 \sigma)$ flash-like 
signal. This is typical for solar particle events in which the gamma-ray emission precedes the low-energy particles by a few minutes. Even so, the hardness ratio $\left(\Delta H R_{500}=+0.5 \sigma\right)$, which is expected to be significantly increased in this case, is not constraining enough. The expected Veto-to-ACS ratio (response) is met, but the ISGRI-to-ACS ratio is off by one order of magnitude, so that the true origin is questionable. Three events might either be too short (950 s at day $-6.0(\mathrm{~h}-144.5), 260 \mathrm{~s}$ at day $-9.5(\mathrm{~h}-227.4))$ or too weak $\left((6.5 \pm 1.7) \times 10^{-3} \mathrm{ph} \mathrm{cm}^{-2} \mathrm{~s}^{-1}\right.$ at $-9.4(\mathrm{~h}-225.5)$, viz. $5.7 \mathrm{kpc}$ distance; SPI $3 \sigma$ upper limit in the 70-520 keV band: $<0.017 \mathrm{ph} \mathrm{cm}^{-2} \mathrm{~s}^{-1}$ ) if the nova model was correct within a factor of three. However, especially the candidate at $\mathrm{h}-227.4$ before the optical maximum attracts attention because it is the only event for which the response is exactly met. Although the gamma-ray light curve is not nova-like, this short peak may only be "the tip of the iceberg", so that most of the photons are either drowned in the background, do not escape, or are not produced, and the nova flash only leaks for several minutes. Including the systematic uncertainties as described above, the flux for this event would be between $(11 \pm 7) \times 10^{-3} \mathrm{ph} \mathrm{cm}^{-2} \mathrm{~s}^{-1}$ (ACS, model-dependent) and $(200 \pm 150) \times 10^{-3} \mathrm{ph} \mathrm{cm}^{-2} \mathrm{~s}^{-1}$ (model-independent, for $260 \mathrm{~s}$ ).

In general, none of the six signal excesses during the weeks before the optical outburst of V5668 can be claimed to be clearly due to the gamma-ray flash of explosive burning. On the other hand, the cases for origins other than the nova (or X-ray transients in general) are also only weak.

\section{Summary, discussion, and conclusions}

\subsection{Nucleosynthesis ejecta}

We report an analysis of INTEGRAL gamma-ray observations of Nova Sgr 2015 No. 2 (V5668 Sgr). Novae are expected to produce significant amounts of ${ }^{7} \mathrm{Be}$. This large mass was detected for the first time for V5668 Sgr by observations of Be II lines in UV wavelengths. Although the ${ }^{7}$ Be II doublet at $313.0583 \mathrm{~nm}$ and $313.1228 \mathrm{~nm}$, respectively, has only an isotopic shift of $\Delta \lambda=-0.161 \AA$ with respect to the ${ }^{9} \mathrm{Be}$ II doublet (Yan et al. $2008)$, the high-resolution spectra from HDT $(R \approx 50000$; cf. Tajitsu et al. 2016, Subaru Telescope) or UVES $(R \approx 100000$, cf. Molaro et al. 2016, VLT) can easily distinguish between the two isotopes for narrow components. There are also other lines from iron-peak elements, such as Cr II or Fe II, which could contaminate the ${ }^{7} \mathrm{Be}$ II measurements but which can also clearly be identified as such. Molaro et al. (2016) estimate the possible contamination of the equivalent width of ${ }^{7} \mathrm{Be}$ II absorption to about $3.5 \%$.

However, the absolute ${ }^{7} \mathrm{Be}$ mass estimates may be more uncertain, and given our limits, it is interesting to check how much larger it could be: Tajitsu et al. (2016) and Molaro et al. (2016) estimate the mass fraction of ${ }^{7} \mathrm{Be}$, following Tajitsu et al. (2015) and Spitzer (1998), by comparing the equivalent widths of a reference element to the ${ }^{7} \mathrm{Be}$ II doublet. Here, the authors used $\mathrm{Ca}$, which is not a nova product, in particular the $\mathrm{Ca}$ II $\mathrm{K}$ line at $393.3 \mathrm{~nm}$. The conversion of the equivalent widths to the respective column densities only works if the lines are not saturated and fully resolved. In addition, the covering factor of the nova shell should not be a strong function of wavelength, as otherwise the Ca II K line could be intrinsically absorbed more strongly or weakly. The compared isotopes must be in the same ionisation state to infer the column density ratios, which are then also the relative elemental abundance. This seems to be true since no doubly ionised or neutral lines for $\mathrm{Ca}$ have been found (Tajitsu et al. 2016; Molaro et al. 2016). Once the abundance ratio $X\left({ }^{7} \mathrm{Be}\right) / X\left({ }^{40} \mathrm{Ca}\right)$ has been determined, an assumption on the $\mathrm{Ca}$ abundance delivers the ${ }^{7} \mathrm{Be}$ abundance in the nova ejecta. Here, the authors assume a solar $\mathrm{Ca}$ abundance, which might underestimate the ${ }^{7} \mathrm{Be}$ abundance by $\approx 30 \%$, due to the abundance gradient in the Milky Way (Cescutti et al. 2007). The ejected ${ }^{7} \mathrm{Be}$ mass was then estimated by Molaro et al. (2016) by assuming a canonical ejected mass of $\approx 10^{-5} M_{\odot}$. In general, the ejected mass may range between $10^{-7}$ and $10^{-3} M_{\odot}$ for $\mathrm{CO}$ novae (Bode \& Evans 2008). Banerjee et al. (2016) estimated a gas ejecta mass of $2.7-5.4 \times 10^{-5} M_{\odot}$, based on a canonical gas-to-dust ratio between 100 and 200, and their measured dust mass of $2.7 \times 10^{-7} M_{\odot}$. The authors assumed a distance to V5668 of $2 \mathrm{kpc}$ in their calculations, so that the dust mass and hence the gas mass, normalised to our $1.6 \mathrm{kpc}$ assumption, may be about $40 \%$ smaller. Altogether, the total ejected mass may be a factor of a few (2-5) larger than canonically expected. This would then also lead to an increase in the ejected ${ }^{7} \mathrm{Be}$ mass to a few $10^{-8} M_{\odot}$. If the ${ }^{7} \mathrm{Be}$ mass was indeed $2 \times 10^{-8} M_{\odot}$, this would be in tension with our derived upper limits on the mass if the distance of $1.6 \mathrm{kpc}$ was correct.

With a half-life time of $\approx 53 \mathrm{~d}$, the radio-isotope ${ }^{7} \mathrm{Be}$ is decaying via electron capture to an excited state of ${ }^{7} \mathrm{Li}$, which de-excites by the emission of a gamma-ray photon at $478 \mathrm{keV}$. Using the spectrometer SPI aboard INTEGRAL, we searched for ${ }^{7} \mathrm{Be}$-line emission during the observations of V5668, which covered several weeks around the nova's optical maximum. From high spectral resolution as well as temporal (light curve) analysis, we found no significant excess in the energy region of interest. We provide $3 \sigma$ upper limits on the $478 \mathrm{keV}$ line flux of $8.2 \times 10^{-5} \mathrm{ph} \mathrm{cm}^{-2} \mathrm{~s}^{-1}$, which can be converted to an upper limit on the ejected ${ }^{7} \mathrm{Be}$ mass of $M_{3 \sigma}^{7 \mathrm{Be}}<1.6 \times 10^{-8} M_{\odot}$. This, however, is based on uncertain distance estimates of $1.6 \mathrm{kpc}$. Assuming an ejected mass as derived by Molaro et al. (2016), we can constrain the distance to V5668 to be further away than $d_{3 \sigma}^{7 \mathrm{Be}}>1.1 \mathrm{kpc}$. Considering the detection of high-energy gamma rays in the $\mathrm{GeV}$ range for V5668 Sgr, the fluxes around the 478 and $1275 \mathrm{keV}$ lines may also have an underlying continuum from shock-accelerated particles. The estimated flux at both lines would be of the order $10^{-8}-10^{-7} \mathrm{ph} \mathrm{cm}^{-2} \mathrm{~s}^{-1}$, following the description of Metzger et al. (2015). Even though this is below the sensitivity limit of SPI, and would only contribute less than $1 \%$ to our upper limits, this effect is already accounted for in our derivation. Because approximated Gaussian line shapes and not only the flux values themselves are used, the line flux limits do not depend on the continuum below.

Nova Sgr 2015 No. 2 was identified as a CO nova, and thus little to no ${ }^{22} \mathrm{Na}$ is expected to be produced and ejected. A gamma-ray line at $1275 \mathrm{keV}$ would reveal the presence of ${ }^{22} \mathrm{Na}$, which is not seen in our analysis $\left(F_{3 \sigma}^{22 \mathrm{Na}}<7.6 \times\right.$ $10^{-5} \mathrm{ph} \mathrm{cm}^{-2} \mathrm{~s}^{-1}$ ).

\subsection{Burst-like emission}

Explosive nucleosynthesis in novae is accompanied by burst-like gamma-ray emission from short-lived isotopes. The $\beta^{+}$decay of these isotopes is expected to be followed by positron annihilation in the nova cloud, leading to a strong annihilation line at $511 \mathrm{keV}$ and continuum down to $\approx 20-30 \mathrm{keV}$, depending on the conditions in the nova. Although this signal would be expected to be of the order of $0.1 \mathrm{ph} \mathrm{cm}^{-2} \mathrm{~s}^{-1}$ at $1 \mathrm{kpc}$ in the $70-520 \mathrm{keV}$ band, i.e. measurable in the SPI-ACS, it has never been observed because it is expected to occur about one week before the optical maximum of the nova. Hence, it may only be seen by chance or by a 
retrospective analysis of large data sets. The time of this gammaray flash is also uncertain, and may range and vary between 2 and 10 days before the optical maximum of a nova. SPI was not pointed to V5668 during the interesting time of the gamma-ray flash. But the INTEGRAL satellite with its main instruments and veto-shields has an almost omni-directional response. Therefore, we performed a search in the SPI-ACS data, using a nova light curve model. Our search found 23 candidate events with significances above $3.9 \sigma$, of which we identify six as possibly being associated with V5668, based also on the directional INTEGRAL response. However, all six excess signals lack strong evidence of really originating from the nova, and all but one would suggest distances of more than $3 \mathrm{kpc}$ (see discussion about positrons below).

Based on temporal, spectral, and directional information from multiple instruments aboard INTEGRAL, we illustrated a way of searching for X-ray transient features in archival data. Our search is similar to GRB analyses (e.g. Rau et al. 2005), but is augmented further by the combined use of energy- and angular-responses of the INTEGRAL veto-systems and main instruments. To date, the INTEGRAL archive comprises $15 \mathrm{yr}$ of data. The estimated galactic-wide nova rate is $50_{-23}^{+31} \mathrm{yr}^{-1}$, and the local nova rate may range between 0.1 and $0.5 \mathrm{kpc}^{-3} \mathrm{yr}^{-1}$ (Shafter 2017), so that tens of novae could be expected to be hidden in the current $15 \mathrm{yr}$ of INTEGRAL data. A thorough retrospective search for X-ray transient features in the INTEGRAL satellite's veto-systems might reveal an entire family of unobserved/unrecognised sources.

While model calculations provide estimates of how much material is produced and ejected, the true conditions shortly after explosive burning are uncertain. The short gamma-ray flash is believed to originate from the injection of positrons from the $\beta^{+}$decay of, predominantly, ${ }^{13} \mathrm{~N}(\tau=14.4 \mathrm{~min})$ and ${ }^{18} \mathrm{~F}$ ( $\tau=158.4 \mathrm{~min}$ ) into the expanding envelope, where they annihilate with electrons and produce the $511 \mathrm{keV}$ annihilation line and a continuum below. At this time, the envelope must be transparent enough to allow the gamma rays to escape, as otherwise no emission would be seen, and the expanding nova cloud would heat up by the absorption of these gamma rays. Although ${ }^{13} \mathrm{~N}$ and ${ }^{18} \mathrm{~F}$ are short-lived, it might also be possible that their decay positrons escape from the nova in large amounts. The positron escape fraction, $f_{\text {esc }}$, could be added as a free parameter, similar to supernovae (e.g. Milne et al. 1999), which would allow diagnostics in the galactic-wide positron puzzle.

The strongest persistent and diffuse soft gamma-ray signal is the $511 \mathrm{keV}$ line from electron-positron annihilation, presumably in the interstellar medium of the Milky Way. The morphology of the emission and the origin of the positrons are probably decoupled because 1) there are more sources to explain the amount of positrons than are actually seen, and 2) the positrons annihilate at rest, which involves a significant deceleration (i.e. kinetic energy loss) from relativistic energies to less than $1 \mathrm{keV}$. This requires several $100 \mathrm{pc}$ propagation distances in typical interstellar medium conditions (e.g. Alexis et al. 2014). Which sources contribute to what extent is only known very roughly (see Prantzos et al. 2011; Siegert 2017, for a review and a global measurement-based discussion, respectively). Based on the apparent non-detection of V5668, novae could add to the reservoir of longer-lived ${ }^{5}$ positrons in the Galaxy if the escape is larger than predicted. Depending on the nova type, of the order $10^{-7}-10^{-3} M_{\odot}$ of material may be ejected (e.g. José \&

\footnotetext{
5 Longer-lived here means no prompt annihilation in the nova itself, but annihilation 0.01-10 Myr later in the interstellar medium.
}

Hernanz 1998; Starrfield et al. 1998). The mass fractions of the dominant positron producers ${ }^{6}{ }^{13} \mathrm{~N}$ and ${ }^{18} \mathrm{~F}$ is of the order $10^{-3}$ and $10^{-4}$ (José et al. 2001, 2003), respectively, so that $10^{-8}-10^{-7} M_{\odot}$ of ${ }^{13} \mathrm{~N}$ and $10^{-9}-10^{-8} M_{\odot}$ of ${ }^{18} \mathrm{~F}$ are created. The decay modes for both isotopes are nearly $100 \%$ positron emission, so that in total $10^{48}-10^{49}$ positrons are created per nova event. Considering the global nova rate, the average number of positrons created by the population of novae in the Milky Way is $(0.9-25.8) \times 10^{42} \times f_{\text {esc }} \mathrm{e}^{+} \mathrm{s}^{-1}$, where $f_{\text {esc }}$ can range between 0 and 1. For example, if all positrons escape, this would make novae the dominant positron producers in the Galaxy. On the one hand, according to Gomez-Gomar et al. (1998), a $100 \%$ escape would be in strong tension with simulations. On the other hand, a 1-10\% escape, as could be suggested for V5668, would contribute to about $1 \%$ of the total required positron production rate to explain the $511 \mathrm{keV}$ emission in the Milky Way (Siegert et al. 2016).

In this work, we showed that INTEGRAL/SPI is capable of detecting a broad ( $8 \mathrm{keV}, \mathrm{FWHM}){ }^{7} \mathrm{Be}$ line at $478 \mathrm{keV}$ from classical novae up to a distance of $\approx 800 \mathrm{pc}$ with $5 \sigma$ significance for an observation time of $1 \mathrm{Ms}$, starting at the visual maximum of the nova. This is derived from tight upper limits on the expected ${ }^{7} \mathrm{Be}$ line flux at $478 \mathrm{keV}$ from the nova V5668 Sgr. In addition, we show that retrospective searches in archival INTEGRAL data can return valuable information for studies of X-ray transients. During the ongoing INTEGRAL mission, at least one such nova event could be expected.

Acknowledgements. This research was supported by the German DFG cluster of excellence "Origin and Structure of the Universe". The INTEGRAL/SPI project has been completed under the responsibility and leadership of CNES; we are grateful to ASI, CEA, CNES, DLR, ESA, INTA, NASA, and OSTC for support of this ESA space science mission. LD and MH acknowledge support from the Spanish MINECO grant ESP2015-66134-R and FEDER funds. J.J. acknowledges support from the Spanish MINECO through grant AYA2014-59084-P, the EU FEDER funds, and the AGAUR/Generalitat de Catalunya grant SGR0038/2014. S.S. acknowledges partial support from NSF, NASA, and HST grants to ASU. T.S. thanks Francesco Berlato for Fermi/LAT analysis of the candidate events.

\section{References}

Ackermann, M., Ajello, M., Albert, A., et al. 2014, Science, 345, 554 Alexis, A., Jean, P., Martin, P., \& Ferrière, K. 2014, A\&A, 564, A108 Banerjee, D. P. K., Ashok, N. M., Venkataraman, V., \& Srivastava, M. 2015, ATel, 7303

Banerjee, D. P. K., Srivastava, M. K., Ashok, N. M., \& Venkataraman, V. 2016, MNRAS, 455, L109

Bode, M. F., \& Evans, A. 2008, Classical Novae, 2nd edn. (Cambridge: Cambridge University Press)

Cash, W. 1979, ApJ, 228, 939

Cescutti, G., Matteucci, F., François, P., \& Chiappini, C. 2007, A\&A, 462, 943 Cheung, C. C., Jean, P., Shore, S. N., \& Fermi Large Area Telescope Collaboration 2013, ATel, 5649

Cheung, C. C., Jean, P., Shore, S. N., et al. 2016, ApJ, 826, 142

Clayton, D. D., \& Hoyle, F. 1974, ApJ, 187, L101

della Valle, M., \& Livio, M. 1995, ApJ, 452, 704

di Leva, A., Gialanella, L., Kunz, R., et al. 2009, Phys. Rev. Lett., 102, 232502

Diehl, R., Siegert, T., Hillebrandt, W., et al. 2014, Science, 345, 1162

Diehl, R., Siegert, T., Greiner, J., et al. 2018, A\&A, 611, A12

Done, C., Gierliński, M., \& Kubota, A. 2007, A\&ARv, 15, 1

Firestone, R. 2003, Overview of Nuclear Data, available online at http: //www . escholarship.org/uc/item/7p80t5po

Gehrz, R. D., Evans, A., Woodward, C. E., et al. 2015, ATel, 7862

6 This is the case for both $\mathrm{CO}$ and ONe novae. In ONe novae, however, of the order of $10^{-8} M_{\odot}$ of ${ }^{22} \mathrm{Na}$ are produced additionally, which is also a $\beta^{+}$decayer, and which contributes to the galactic positron content because ${ }^{22} \mathrm{Na}$ has a half-life time of $2.75 \mathrm{yr}$, i.e. at times when the nova is fully transparent and the ejecta are further away from the white dwarf. 
T. Siegert et al.: Gamma-ray observations of Nova Sgr 2015 No. 2 with INTEGRAL

Gomez-Gomar, J., Hernanz, M., José, J., \& Isern, J. 1998, MNRAS, 296, 913

Gros, M., Tatischeff, V., Kiener, J., et al. 2004, ESA SP, 552, 669

Hays, E., Cheung, T., \& Ciprini, S. 2013, ATel, 5302

Hernanz, M. 2005, in The Astrophysics of Cataclysmic Variables and Related Objects, eds. J.-M. Hameury \& J.-P. Lasota, ASP Conf. Ser., 330, 265

Hernanz, M. 2014, in Stellar Novae: Past and Future Decades, eds. P. A Woudt \& V. A. R. M. Ribeiro, ASP Conf. Ser., 490, 319

Hernanz, M., \& José, J. 2006, New Astron. Rev., 50, 504

Hernanz, M., José, J., Coc, A., \& Isern, J. 1996, ApJ, 465, L27

Hernanz, M., Gómez-Gomar, J., José, J., \& Isern, J. 1997, ESA SP, 382, 47

Izzo, L., Della Valle, M., Mason, E., et al. 2015, ApJ, 808, L14

Jack, D., Robles Pérez, J. d. J., De Gennaro Aquino, I., et al. 2017, Astron. Nachr., 338, 91

Jean, P., Gómez-Gomar, J., Hernanz, M., et al. 1999, Astrophys. Lett. Commun., 38,421

José, J. 2016, Stellar Explosions: Hydrodynamics and Nucleosynthesis (Boca Raton, FL: CRC Press)

José, J., \& Hernanz, M. 1998, ApJ, 494, 680

José, J., Hernanz, M., \& Coc, A. 2001, Nucl. Phys. A, 688, 118

José, J., Hernanz, M., García-Berro, E., \& Gil-Pons, P. 2003, ApJ, 597, L41

José, J., Hernanz, M., \& Iliadis, C. 2006, Nucl. Phys. A, 777, 550

Kiener, J., Gros, M., Tatischeff, V., \& Weidenspointner, G. 2006, A\&A, 445, 725

Leising, M. D., \& Clayton, D. D. 1987, ApJ, 323, 159

Luckas, P. 2016, ATel, 9678

Martin, P., Dubus, G., Jean, P., Tatischeff, V., \& Dosne, C. 2018, A\&A, 612, A38

Metzger, B. D., Hascoët, R., Vurm, I., et al. 2014, MNRAS, 442, 713

Metzger, B. D., Finzell, T., Vurm, I., et al. 2015, MNRAS, 450, 2739

Milne, P. A., The, L.-S., \& Leising, M. D. 1999, ApJS, 124, 503
Molaro, P., Izzo, L., Mason, E., Bonifacio, P., \& Della Valle M. 2016, MNRAS, 463, L117

Page, K. L., Kuin, N. P. M., Osborne, J. P., \& Schwarz, G. J. 2015, ATel, 7953 Parker, P. D., \& Kavanagh, R. W. 1963, Phys. Rev., 131, 2578

Prantzos, N., Boehm, C., Bykov, A. M., et al. 2011, Rev. Mod. Phys., 83, 1001

Rau, A., Kienlin, A. V., Hurley, K., \& Lichti, G. G. 2005, A\&A, 438, 1175

Savchenko, V., Bazzano, A., Bozzo, E., et al. 2017a, A\&A, 603, A46

Savchenko, V., Ferrigno, C., Kuulkers, E., et al. 2017b, ApJ, 848, L15

Schmidt, T. 1957, Z. Astrophys., 41, 182

Schwarzschild, M., \& Härm, R. 1965, ApJ, 142, 855

Seach, J. 2015, Central Bureau Electronic Telegrams, 4080

Shafter, A. W. 2017, ApJ, 834, 196

Siegert, T. 2017, Dissertation, Technische Universität München, Germany. Available at https://mediatum.ub.tum.de/node?id=1340342

Siegert, T., Diehl, R., Khachatryan, G., et al. 2016, A\&A, 586, A84

Spitzer, L. 1998, Physical Processes in the Interstellar Medium (Weinheim, Germany:Wiley VCH Verlag $\mathrm{GmbH}), 335$

Starrfield, S., Truran, J. W., Sparks, W. M., \& Kutter, G. S. 1972, ApJ, 176, 169

Starrfield, S., Truran, J. W., Wiescher, M. C., \& Sparks, W. M. 1998, MNRAS, 296, 502

Tajitsu, A., Sadakane, K., Naito, H., Arai, A., \& Aoki, W. 2015, Nature, 518, 381

Tajitsu, A., Sadakane, K., Naito, H., et al. 2016, ApJ, 818, 191

Ubertini, P., Lebrun, F., Di Cocco, G., et al. 2003, A\&A, 411, L131

Vedrenne, G., Roques, J.-P., Schönfelder, V., et al. 2003, A\&A, 411, L63

Vestrand, W. T., Share, G. H., J. Murphy, R., et al. 1999, ApJS, 120, 409

Winkler, C., Courvoisier, T. J.-L., Di Cocco, G., et al. 2003, A\&A, 411, L1

Yan, Z.-C., Nörtershäuser, W., \& Drake, G. W. F. 2008, Phys. Rev. Lett., 100, 243002

Yoon, S.-C., Langer, N., \& van der Sluys, M. 2004, A\&A, 425, 207 